\title{
Intramyocardial and intracoronary basic fibroblast growth factor in porcine hibernating myocardium: A comparative study
}

\author{
Shankha S. Biswas, MD \\ G. Chad Hughes, MD \\ John E. Scarborough, $\mathrm{MD}^{\mathrm{a}}$ \\ Patrick W. Domkowski, MD, PhDa \\ Luis Diodato, MD \\ Monica L. Smith, BS ${ }^{a}$ \\ Carolyn Landolfo, $\mathrm{MD}^{\mathrm{b}}$ \\ James $\mathrm{E}$. Lowe, $\mathrm{MD}^{\mathrm{a}}$ \\ Brian H. Annex, MD \\ Kevin P. Landolfo, MD
}

From the Divisions of Cardiothoracic Surgery $^{\mathrm{a}}$ and Cardiology, ${ }^{\mathrm{b}}$ Duke University Medical Center, Durham, NC.

Read at the Eighty-first Annual Meeting of The American Association for Thoracic Surgery, San Diego, Calif, May 6-9, 2001.

Funded by the Chiron Corporation, Emeryville, Calif.

Received for publication Feb 7, 2002; revisions requested April 16, 2002; revisions received June 30, 2003; accepted for publication July 10, 2003.

Address for reprints: James E. Lowe, MD, Duke University Medical Center, Box 3954, Durham, NC 27710 (E-mail: lowe0004@mc.duke.edu).

J Thorac Cardiovasc Surg 2004;127:34-43 $0022-5223 / \$ 30.00$

Copyright $\odot 2004$ by The American Association for Thoracic Surgery

doi:10.1016/j.jtcvs.2003.07.003

Objective: Therapeutic angiogenesis is an alternative method of revascularization for end-stage coronary artery disease. We determined the effects of intramyocardial and intracoronary basic fibroblast growth factor 2 on myocardial blood flow and function in a porcine model of hibernating myocardium.

Methods: Twenty-four mini-swine with $90 \%$ left circumflex artery stenosis and documented hibernating myocardium by positron emission tomography and dobutamine stress echocardiography were randomized to intramyocardial basic fibroblast growth factor 2 at $0.6 \mu \mathrm{g} / \mathrm{kg}$ (mid-dose, $\mathrm{n}=6,30$ injections/animal), $6 \mu \mathrm{g} / \mathrm{kg}$ (high-dose, $\mathrm{n}=6,30$ injections/animal), or intramyocardial vehicle control $(\mathrm{n}=6)$. The intracoronary group received $6 \mu \mathrm{g} / \mathrm{kg}$ basic fibroblast growth factor $2(n=6)$ into the right and left circumflex artery coronary arteries. Positron emission tomography and dobutamine stress echocardiography were repeated at 1 and 3 months.

Results: In the vehicle group, normalized left circumflex artery myocardial blood flow was $0.74 \pm 0.04$ at 1 month and $0.75 \pm 0.07$ at 3 months compared with 0.68 \pm 0.03 at baseline. In the intracoronary group, myocardial blood flow was $0.71 \pm$ 0.03 at 1 month and $0.72 \pm 0.04$ at 3 months compared with $0.67 \pm 0.04$ at baseline. In the mid group, myocardial blood flow was $0.73 \pm 0.06$ at 1 month and $0.85 \pm$ 0.05 at 3 months $(P<.001)$ compared with $0.67 \pm 0.04$ at baseline. In the high group, myocardial blood flow was $0.81 \pm 0.06$ at 1 month and $0.83 \pm .04$ at 3 months $(P=.03)$ compared with $0.71 \pm 0.02$ at baseline. No significant improvements in ischemia were demonstrated in any of the groups by dobutamine stress echocardiography at 1 or 3 months.

Conclusions: In porcine hibernating myocardium, intramyocardial basic fibroblast growth factor 2 significantly improved regional myocardial blood flow 3 months after treatment. There was no significant change in function in any of the 4 groups. These data suggest that intramyocardial dosing of basic fibroblast growth factor 2 $(0.6 \mu \mathrm{g} / \mathrm{kg})$ may be an optimal dose for improving perfusion in the treatment of end-stage coronary artery disease. 


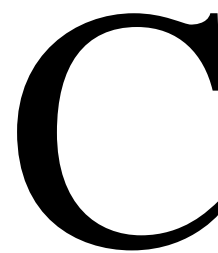

oronary artery disease continues to be the major cause of mortality and morbidity in the Western world despite continued advances in the treatment of ischemic heart disease. Nearly 13.5 million Americans suffer from coronary artery disease; a growing number of patients are unamenable to standard therapy such as coronary artery bypass grafting or percutaneous coronary angioplasty because of diffuse disease and poor runoff vessels on angiography, a lack of available conduits, high operative risk, or various combinations of these factors. ${ }^{1}$ An estimated $5 \%$ to $15 \%$ of patients undergoing coronary angiography may constitute this population. ${ }^{2}$ Therapeutic angiogenesis may provide a novel approach to the treatment of myocardial ischemia for these patients.

Therapeutic angiogenesis seeks to treat disorders of inadequate tissue perfusion through growth and proliferation of new blood vessels. ${ }^{3}$ Angiogenesis is defined as the formation of new blood vessels from preexisting vessels by the process of cellular outgrowth. This process involves a complex series of events including the following processes: the breakdown of existing extracellular matrix; smooth muscle and endothelial cell migration, adhesion, and proliferation; the formation of new vascular structures; and the deposition of new extracellular matrix. ${ }^{4}$ Basic fibroblast growth factor (bFGF) has been studied both experimentally and clinically utilizing various routes of delivery and doses for the treatment of ischemic heart disease. bFGF is a single-chain, heparin-binding, 16-kD 154 amino acid peptide with both mitogenic and angiogenic properties. However, angiogenic growth factors have been associated with pathologic angiogenesis and tumorigenesis. ${ }^{5-10}$ The long-term efficacy of a safe low dose of bFGF in hibernating myocardium remains to be established.

A series of investigators have examined the effects of recombinant bFGF in both swine and canine species using an ameroid constrictor model. ${ }^{11-15}$ The longest period between treatment with bFGF and follow-up assessment of perfusion and function was 8 weeks. Longer study periods have been difficult to achieve as the ameroid model is limited by the eventual development of a collateral circulation to the ischemic areas of myocardium. Therefore, the purpose of our study was to determine the longer-term effects ( 3 months) of basic FGF-2 using either intramyocardial and intracoronary (IC) delivery. To examine the longterm effects of this exogenous protein on myocardial perfusion and function, we used a porcine model of hibernating myocardium previously developed and validated in our laboratory.

\section{Methods}

A total of 24 adult male mini-swine (25-35 kg) were used. Animals were obtained from Harlan-Sinclair (Indianapolis, Ind) and housed under standard conditions and fed a regular diet. The Animal Care and Use Committee of Duke University approved all procedures and protocols. Animals received humane care in compliance with the Principles of Laboratory Animal Care formulated by the $\mathrm{Na}$ tional Society for Medical Research and the Guide for the Care and Use of Laboratory Animals prepared by the Institute of Laboratory Animal Resources and published by the National Institutes of Health (NIH publication 85-23, revised 1996).

\section{Partial Coronary Occlusion Model}

All animals underwent induction of anesthesia with the combination tiletamine/zolazepam ( $5 \mathrm{mg} / \mathrm{kg}$ intramuscularly). Orotracheal intubation was performed and anesthesia maintained with isoflurane $(2 \%)$ while the animals were mechanically ventilated. Continuous electrocardiographic and pulse-oximetric monitoring was used throughout the procedure to ensure a stable cardiac rhythm and adequate oxygenation. Ampicillin (1 g IV), gentamicin (80 mg IV), and bretylium tosylate $(5 \mathrm{mg} / \mathrm{kg}$ IV) were given preoperatively. Initial muscle relaxation was obtained with a single injection of pancuronium bromide ( $2 \mathrm{mg}$ IV). Under sterile conditions, a left anterolateral thoracotomy was performed through the third intercostal space. The pericardium was incised longitudinally, and the left atrial appendage was retracted to allow exposure of the left circumflex coronary artery (LCX). The proximal LCX was dissected free to allow placement of a hydraulic occluder and 2-mm ultrasonic flow probe (Transonic Systems, Inc, Ithaca, NY) around the vessel. The flow probe was placed distal to the occluder to record downstream flow through the LCX. The pericardium was closed. The occluder and flow probe were then exteriorized through a separate stab incision. A 20-French chest tube was placed and the wound closed in layers. The chest tube was removed at the conclusion of the procedure. The animals were fitted with specially designed jackets (Lomir Biomedical, Quebec, Canada) to protect and allow easy access to their externalized hardware.

Three days postoperatively the animals were lightly sedated with the combination tiletamine/zolazepam $(5 \mathrm{mg} / \mathrm{kg}$ intramuscularly) and the occluder inflated to reduce resting blood flow in the LCX downstream from the occluder to $10 \%$ of baseline as assessed using the implanted flow probe. Once flow was stable for 5 minutes, the animals were returned to their cages. Flow was rechecked the following day (postoperative day 4) and the occluder adjusted as needed to maintain a $90 \%$ flow reduction. This generally required additional tightening due to capacitance of the occluder. Flow was then rechecked again the following day (postoperative day 5) to assure continued occlusion. No additional occluder manipulation was performed after the second flow check. Animals were then kept in this low-flow state for the duration of the experiment. Animals were medicated with aspirin $(650 \mathrm{mg}$ po every day) throughout the entire experiment.

\section{Positron Emission Tomography and Dobutamine Stress Echocardiography}

Animals underwent baseline positron emission tomography (PET) and dobutamine stress echocardiography (DSE) to characterize myocardial blood flow, metabolism, and function as previously described. ${ }^{16}$ This was performed 30 days postocclusion (postoperative day 34). PET scans were interpreted as showing hibernat- 


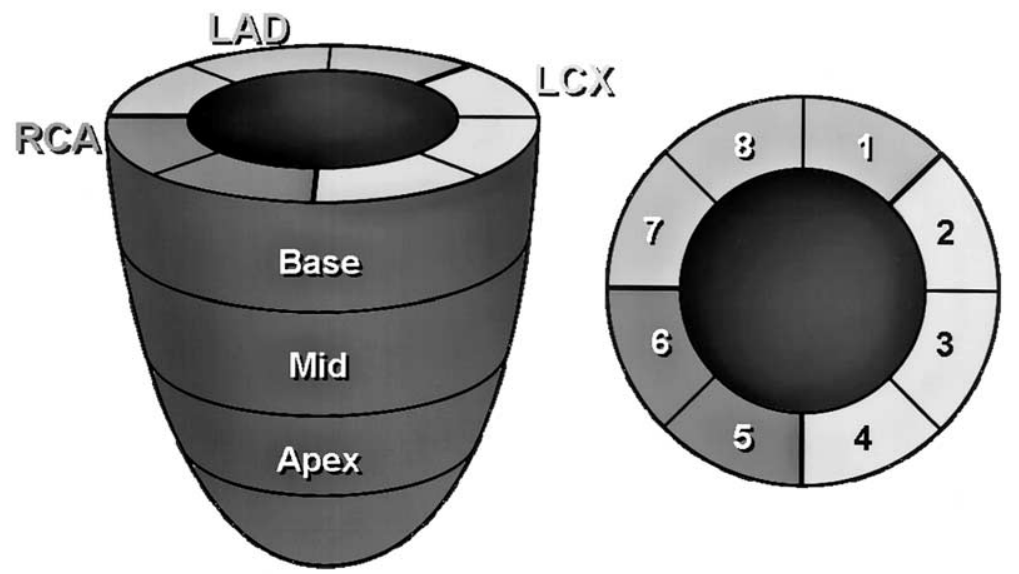

Figure 1. Diagram of the 8 sectors used for comparing quantitative measurements of myocardial blood flow and glucose utilization in the $\mathbf{3}$ short-axis slices (basal, mid, and apical). Sectors $\mathbf{2}$ through 4 (lateral and posteroinferior walls of the left ventricle) were considered as representing myocardium within the left circumflex distribution. Sectors 7 and 8 served as the nonischemic control regions.

ing myocardium if a flow deficit was noted in the lateral and posteroinferior walls of the left ventricle supplied by the LCX accompanied by normal or increased glucose utilization in these same regions (both as compared to the nonischemic septum). ${ }^{17}$ Using DSE, viability in the lateral and posteroinferior walls of the left ventricle was defined as an improvement in systolic wall thickening with low-dose dobutamine in myocardial regions with severe hypocontractility at rest. ${ }^{18}$ Viable segments were considered ischemic if systolic wall motion deteriorated with stress (biphasic response). ${ }^{19}$

After an overnight fast, dynamic PET emission imaging of the heart was performed at rest for 20 minutes following a 30 -second IV infusion of ${ }^{13} \mathrm{~N}$-ammonia $(15-20 \mathrm{mCi})$. A 40-minute delay followed for decay of ${ }^{13} \mathrm{~N}$ activity. Then dynamic imaging of the heart for 60 minutes was performed following a 30-second infusion of ${ }^{18} \mathrm{~F}$-fluorodeoxyglucose (FDG; $10 \mathrm{mCi}$ ). All emission images were corrected for photon attenuation using a transmission scan. Time-activity curves of tracer concentration in the left ventricular myocardium were obtained from short-axis views averaged over 8 sectors in 3 short-axis slices (basal, mid, and apical, Figure 1). Previously validated compartmental modeling techniques were applied to the time-activity curves to obtain regional estimates of myocardial blood flow ( $\mathrm{mL} / \mathrm{g} / \mathrm{min})$ and glucose utilization (nmol/g/min). ${ }^{20,21}$ A "lumped constant" of 1.0 was assumed for the calculation of glucose utilization from fractional FDG uptake rate. ${ }^{22}$ During the PET scans, animals were maintained under general anesthesia and mechanically ventilated as described above. PET data was interpreted in a blinded manner both qualitatively by a physician experienced reading cardiac PET scans and quantitatively using absolute values for blood flow $(\mathrm{mL} / \mathrm{g} / \mathrm{min})$ and glucose utilization ( $\mathrm{nmol} / \mathrm{g} / \mathrm{min})$. Absolute values of myocardial blood flow and glucose utilization were compared within each individual PET study to determine whether viability and ischemia were present in the LCX distribution. For each study, sectors representing the anterior septum (left anterior descending coronary distribution) were used as the normal reference segments.

\section{DSE}

DSE was performed in 3-minute stages with incremental doses of dobutamine beginning with $5 \mu \mathrm{g} / \mathrm{kg} / \mathrm{min}$ and increasing to 40 $\mu \mathrm{g} / \mathrm{kg} / \mathrm{min}$. Based on a standard 16-segment model, wall motion was graded as $1=$ normal, $2=$ hypokinetic (reduced systolic wall thickening), 3 = akinetic (absent systolic wall thickening), or $4=$ dyskinetic (outward systolic wall motion). Regional wall motion score index (WMSI) was calculated at rest, low dose, and peak stress. Animals were sedated with the combination tiletamine/zolazepam ( $5 \mathrm{mg} / \mathrm{kg}$ intramuscularly) prior to DSE. A single cardiologist blinded to the clinical status of the miniswine and with expertise in stress echocardiography interpreted the echoes. ${ }^{16}$

\section{Growth Factor Delivery}

After partial left circumflex arterial occlusion and subsequent documentation of myocardial hibernation by PET and DSE, each animal was randomized to receive recombinant human bFGF or vehicle via 1 of 4 groups: high-dose intramyocardial (IM) bFGF (6 $\mu \mathrm{g} / \mathrm{kg})$, mid-dose IM bFGF (0.6 $\mu \mathrm{g} / \mathrm{kg})$, IC bFGF ( $6 \mu \mathrm{g} / \mathrm{kg})$, or IM vehicle injection. The second operation was performed within 3 days of completion of baseline studies. Anesthesia, preoperative medications, and intraoperative monitoring were as described as above. Baseline systolic blood pressure, heart rate, serum chemistries, and bFGF-2 serum levels were measured immediately after the induction of general anesthesia. Intramyocardial bFGF or vehicle was administered at the above doses to treat the entire LCX region via $300.1-\mathrm{mL}$ intramyocardial injections at $1-\mathrm{cm}$ intervals. Care was taken to avoid all epicardial and intramyocardial vessels. Hemostasis was obtained by manual compression. The occluder and flow probe were left intact. The pericardium was left widely open. Repeat systolic blood pressure and heart rate were measured immediately after bFGF or vehicle injection. The wound was closed as described previously. The animals were kept under general anesthesia for 2 hours after intramyocardial injection and 
repeat measurements of serum chemistry, heart rate, and systolic blood pressure were repeated. Continuous LCX occlusion was confirmed postoperatively by weekly flow monitoring with the flow probe.

IC bFGF was delivered to 6 animals with hibernating circumflex myocardium using a sterile femoral artery cut-down and the Judkins technique. The animals were under general anesthesia during this procedure. Heparin $70 \mathrm{U} / \mathrm{kg}$ was given intravenously immediately prior to the femoral artery cut-down. Systolic blood pressure, heart rate, serum chemistries, and bFGF serum levels were collected immediately after the femoral arterial sheath was placed. The coronary artery anatomy was reassessed prior to bFGF delivery. Near total occlusion of the LCX with minimal collateralization was demonstrated with antegrade flow down the LCX artery. Six $\mu \mathrm{g} / \mathrm{kg}$ bFGF was divided into 2 injections and delivered into the left circumflex artery over a 10-minute period, followed by the right coronary injection over a 10-minute period. Systolic blood pressure and heart rate were measured in between the LCX and right coronary artery injections. Next, the groins were closed and the animals kept under general anesthesia for 2 hours, after which measurements of serum chemistry, systolic blood pressure, and heart rate were repeated.

\section{Follow-up PET and DSE}

One and 3 months after the second procedure, the bFGF- and vehicle-treated animals had repeat PET and DSE as described previously. To allow comparisons between studies performed at baseline and 1 and 3 months postoperatively and to correct for known interstudy variability of absolute values of myocardial blood flow obtained by PET, ${ }^{17,23}$ the data were normalized using previously described techniques. ${ }^{17}$ For each study, sectors representing the anterior septum (sectors 7 and 8 in Figure 1) were used as the normal reference segments. ${ }^{13} \mathrm{~N}$-ammonia activity in the sectors representing the LCX distribution (sectors 2 to 4 in Figure 1) was then expressed as a percentage of the activity measured in the reference segments.

\begin{abstract}
Animal Death
Three months after the second procedure, the animals were killed under general anesthesia. Cardiac catheterization was performed via the Judkins technique and the hearts were explanted through a median sternotomy. Sections of the left ventricle were frozen in liquid nitrogen, as well as placed in sucrose solution for alkaline phosphatase staining for vascular density analysis.
\end{abstract}

\section{Vascular Density Analysis}

To further describe differences in neovascularization in treated ischemic myocardium, we made a comprehensive comparison of neovessel density in these regions. Using previously described techniques, ${ }^{24}$ vascular density was measured by endogenous endothelial alkaline phosphatase staining on unfixed frozen sections of the left ventricles in the bFGF and IM vehicle groups. Vascular density was measured by counting. From each animal, samples were examined from the LCX ischemic distribution. Six random high-power $(200 \times)$ fields were examined per sample and quantitated using an NIH imaging program (version 1.62).
TABLE 1. Normalized myocardial perfusion by positron emission tomography for left circumflex artery distribution preoperatively and 1 and 3 months postoperatively following treatment

\begin{tabular}{lccc}
\hline & \multicolumn{3}{c}{ Perfusion ratio } \\
\cline { 2 - 4 } Treatment group & Baseline & $\begin{array}{c}\text { 1 month } \\
\text { posttreatment }\end{array}$ & $\begin{array}{c}\text { 3 months } \\
\text { posttreatment }\end{array}$ \\
\hline High $(\mathrm{n}=6)$ & $0.71 \pm 0.02$ & $0.81 \pm 0.06$ & $0.83 \pm 0.04^{*}$ \\
Mid $(\mathrm{n}=6)$ & $0.67 \pm 0.04$ & $0.73 \pm 0.06$ & $0.85 \pm 0.05^{*}$ \\
IC $(\mathrm{n}=6)$ & $0.67 \pm 0.04$ & $0.71 \pm 0.03$ & $0.72 \pm 0.04$ \\
Vehicle $(\mathrm{n}=6)$ & $0.68 \pm 0.03$ & $0.74 \pm 0.04$ & $0.75 \pm 0.07$
\end{tabular}

$I C$, Intracoronary. Normalized myocardial perfusion = mean blood flow left circumflex distribution $\div$ mean blood flow nonischemic septum, in $\mathrm{mL} / \mathrm{g} /$ $\min$. Values reported as the mean \pm standard error of the mean.

${ }^{*} P=.033$ and $P<.001$ for the high and mid groups, respectively, by 1 -way repeated measures ANOVA.

\section{Statistical Analysis}

Data were analyzed using Sigmastat for Windows version 2.0 (SPSS Inc, Chicago, Ill). All data are presented as the mean \pm standard error of the mean. Baseline and 1- and 3-month PET data was compared within groups using 1-way repeated measures analysis of variance (RM ANOVA) followed by the Tukey method for multiple comparison procedure. DSE regional WMSI in the base and mid portions of the lateral, posterior, and inferior segments of the left ventricle were compared at baseline and 1 and 3 months using 1-way RM ANOVA followed by the Tukey tests for multiple comparison procedure. Vascular density was compared by Kruskal-Wallis 1-way ANOVA on ranks with post hoc Tukey tests. Statistical significance was considered a $P$ value less than .05 .

\section{Results}

All but 2 of the animals enrolled in this study survived to the 3 -month end point. Two animals died 1 month after IC bFGF delivery secondary to sepsis from infected groin wounds. No abnormal adverse events were noted during the entire course of these experiments in those animals that survived the study.

\section{PET}

Analysis of quantitated normalized LCX myocardial blood flow 1 and 3 months following IM vehicle revealed a slight increase in normalized ${ }^{13} \mathrm{~N}$-ammonia accumulation similar to that seen at baseline (Table 1). Resting myocardial blood flow was not significantly improved in the IC and vehicle groups at both 1 and 3 months after treatment $(P=.38$ and .41 , respectively, by 1-way RM ANOVA; Figure 2). However, at 3 months following both high- and mid-dose IM bFGF, there was a significant improvement $(27.8 \% \pm 5.2 \%$ and $16.6 \% \pm 5.0 \%$, respectively) in LCX resting myocardial blood flow (MBF) $(P<.001$ and $P=.033$, respectively by 1-way RM ANOVA), which was not demonstrated at the 1-month time point. 


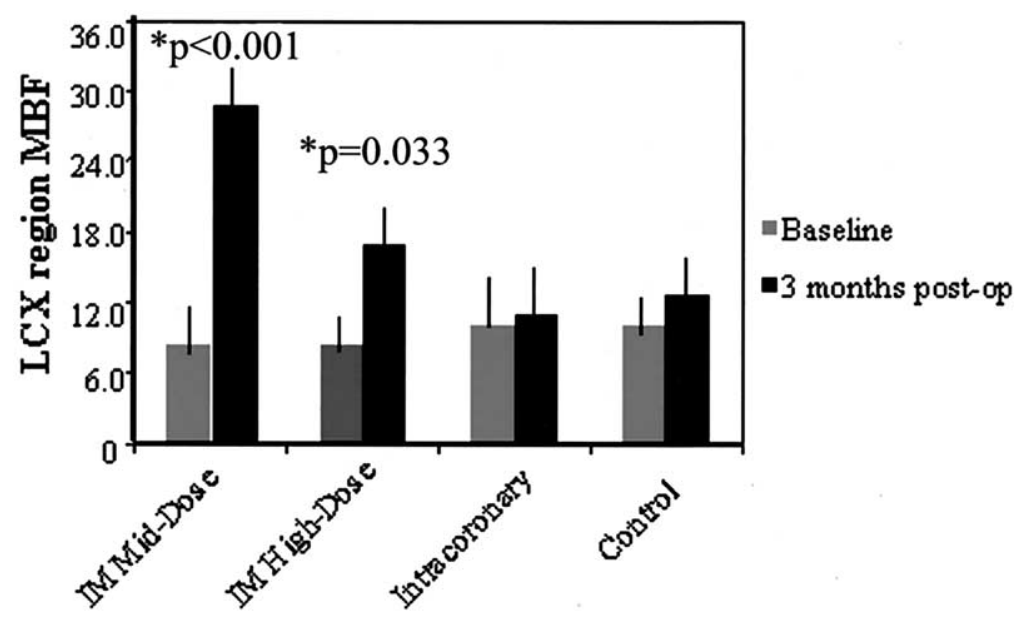

Figure 2. PET perfusion at baseline and 3 months after treatment. The high and mid groups had significantly improved perfusion 3 months after treatment.

TABLE 2. ${ }^{18} \mathrm{~F}$-fluorodeoxyglucose measurements

\begin{tabular}{lcc}
\hline Treatment group & Apex & Mid-base \\
\hline High $(\mathrm{n}=6)$ & $1.72 \pm 0.25$ & $1.50 \pm 0.33$ \\
Mid $(\mathrm{n}=6)$ & $1.84 \pm 0.57$ & $1.48 \pm 0.35$ \\
IC $(\mathrm{n}=6)$ & $1.08 \pm 0.12$ & $3.11 \pm 0.90$ \\
Vehicle $(\mathrm{n}=6)$ & $1.83 \pm 0.28$ & $2.11 \pm 0.71$ \\
\hline
\end{tabular}

$I C$, Intracoronary. Values reported as the mean \pm standard error of the mean.

Table 2 summarizes the FDG accumulations in the 24 animals. There was normal to increased FDG uptake in the area of LCX perfusion abnormality (mid-base regions) when compared with the apical regions, which was consistent with hibernating myocardium in the LCX distribution.

\section{DSE}

Baseline DSE demonstrated severe hypocontractility at rest in the lateral and posteroinferior walls of the left ventricle supplied by the partially occluded LCX coronary artery in all 4 groups (Figure 3). Wall motion in these regions demonstrated a biphasic response of initial improvement followed by deterioration with dobutamine stimulation (Table 3 ), which is consistent with ischemic, viable myocardium in the LCX distribution.

For the IM vehicle control, mid-dose IM bFGF, highdose IM bFGF, and IC bFGF groups at 1 and 3 months following injection, there was no significant reduction in ischemia in either resting or peak stress regional WMSI (Table 3).

\section{Vascular Density}

Quantitative vascular density (arbitrary units) by 1-way ANOVA on ranks demonstrated significantly higher left ventricular neovascularization in the mid group when compared to the high, IC, and vehicle groups $(P<.001$, Table 4) by Kruskal-Wallis 1-way ANOVA on ranks with Tukey tests for multiple comparison procedures (Figure 4). These findings are consistent with the observed qualitative differences and provide further evidence for neovascularization within areas of ischemic, viable myocardium treated with mid-dose IM bFGF.

\section{Chemistry and Hemodynamic Data}

Tables 5, 6, 7 , and 8 summarize the serum chemistry and hemodynamic data, respectively, obtained during the course of the study.

In terms of serum chemistry changes in the high group, only serum glucose levels were significantly different at the 2-hour time point versus baseline $(P=.04$ by 1 -way RM ANOVA). For the mid group and the IM vehicle group, there were no significant changes noted. With respect to hemoglobin, platelets, sodium, $\mathrm{CO}_{2}$, and creatinine at baseline, 2 hours, and 3 months, no significant changes were noted. No significant heart rate changes were observed in either the high, mid, or IC group with respect to control animals. Similarly, systolic blood pressure (SBP) was not significantly altered in any of the treatment arms in any of the measured time points.

\section{Serum bFGF-2 Levels}

Table 9 summarizes serum bFGF-2 levels at baseline and at 3 months after treatment. Only the IC bFGF group had a significantly higher bFGF-2 level at 3 months when compared with baseline $(P=.038$ by 1 -way $\mathrm{RM}$ ANOVA). The high, mid, and vehicle groups had serum bFGF-2 levels that were unchanged from baseline when compared at 3 months. 


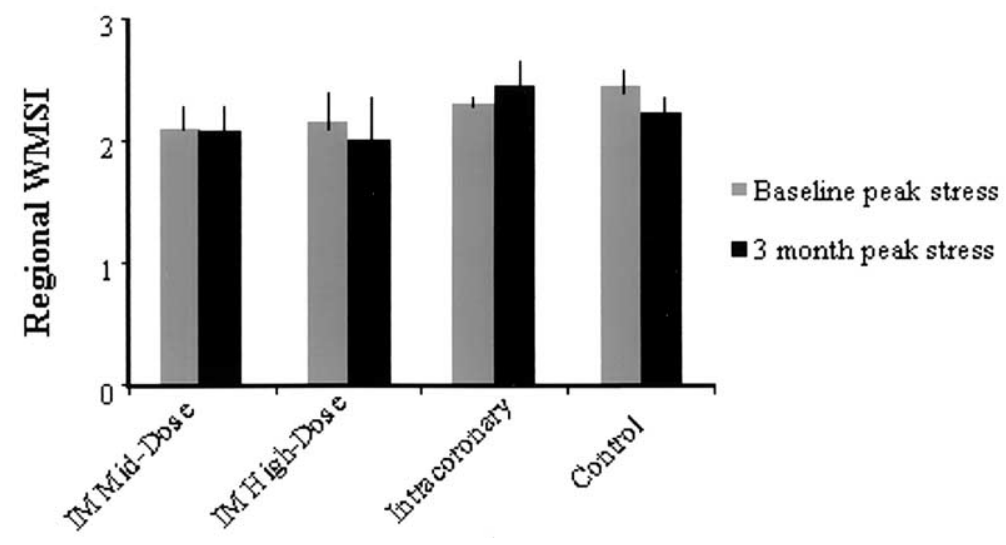

Figure 3. Peak stress function as measured by dobutamine stress echocardiography. No improvement was seen in peak stress function in any of the $\mathbf{4}$ groups as measured by dobutamine stress echocardiography.

TABLE 3. Mean regional wall motion score index preoperatively and 1 and 3 months postoperatively following treatment

\begin{tabular}{lcc}
\hline Treatment & Rest & Peak stress \\
\hline High & & \\
$\quad$ LCX baseline & $2.2 \pm 0.1$ & $2.0 \pm 0.1$ \\
LCX 1 month & $2.1 \pm 0.1$ & $1.8 \pm 0.2$ \\
$\quad$ LCX 3 months & $2.0 \pm 0.1$ & $1.9 \pm 0.2$ \\
Mid & & \\
$\quad$ LCX baseline & $2.1 \pm 0.1$ & $1.8 \pm 0.1$ \\
LCX 1 month & $2.1 \pm 0.1$ & $1.7 \pm 0.2$ \\
LCX 3 months & $2.1 \pm 0.1$ & $1.8 \pm 0.2$ \\
IC & & \\
LCX baseline & $2.2 \pm 0.1$ & $2.0 \pm 0.2$ \\
LCX 1 month & $2.2 \pm 0.1$ & $2.0 \pm 0.2$ \\
LCX 3 months & $2.3 \pm 0.1$ & $2.2 \pm 0.2$ \\
Vehicle & & \\
LCX baseline & $2.3 \pm 0.1$ & $2.0 \pm 0.1$ \\
LCX 1 month & $2.2 \pm 0.1$ & $1.9 \pm 0.2$ \\
LCX 3 months & $2.0 \pm 0.1$ & $2.1 \pm 0.1$ \\
\hline
\end{tabular}

$\overline{L C X}$, Left circumflex coronary artery. Wall motion scores: $1=$ normal; $2=$ hypokinetic; 3 = akinetic; $4=$ dyskinetic. Values reported as the mean \pm standard error of the mean.

\section{Discussion}

In this study, mid-dose bFGF $(0.6 \mu \mathrm{g} / \mathrm{kg})$ improved resting myocardial blood flow by PET 3 months after direct intramyocardial injection. However, no significant improvements were seen in the resting wall motion abnormalities at either mid- or high-dose bFGF as evaluated by DSE. Vascular density analysis revealed significantly higher left ventricular neovascularization following IM mid-dose bFGF when compared with IM high dose $(6 \mu \mathrm{g} / \mathrm{kg})$, intracoronary (6 $\mu \mathrm{g} / \mathrm{kg}$ ) bFGF, and IM vehicle control. Lastly, the serum chemistry and hemodynamic data revealed no adverse effects of mid-dose, high-dose, and IC bFGF-2 over the 3 month time course.
TABLE 4. Vascular density analysis for evidence of neovascularization

Treatment group

Quantitative vascular density

High $(\mathrm{n}=6)$

Mid $(\mathrm{n}=6)$

IC $(\mathrm{n}=6)$

$1561.8 \pm 76.1$

Vehicle $(\mathrm{n}=6)$

$2286.0 \pm 100.5^{*}$

$1821.4 \pm 133.7$

$1789.3 \pm 103.7$

IC, Intracoronary. Quantitative vascular density analysis reported as the mean \pm standard error of the mean, in arbitrary units.

${ }^{*} P=.04$ by 1 -way ANOVA on ranks versus all other groups.

Previous investigators have utilized the ameroid constrictor model for evaluation of angiogenic growth factors. Harada and colleagues ${ }^{11}$ demonstrated in swine that 4 to 5 $\mu \mathrm{g}$ bFGF delivered periadventitially via heparin-alginate beads improved coronary flow and reduced infarct size with prevention of pacing-induced hemodynamic deterioration up to 8 weeks after surgery. Unger and associates ${ }^{13}$ in 1994 utilized a similar ameroid constrictor model in dogs and demonstrated using $110 \mu \mathrm{g}$ bFGF via intracoronary delivery distal to the occluded left circumflex artery enhanced maximal collateral blood flow 4 weeks after treatment. In 1997, Lopez and colleagues ${ }^{12}$ studied swine using 10 or $100 \mu \mathrm{g}$ bFGF; at 8 weeks follow-up they demonstrated higher angiographic collateral index, improved thrombosis in myocardial infarction (TIMI) flow scores and coronary flow compared with controls, and improved regional and global left ventricular function with significantly better preservation of regional wall motion in the $100-\mu \mathrm{g}$ group. Using a swine ameroid model, Laham and colleagues demonstrated significant increases in left-to-right angiographic collaterals and left circumflex artery blood flow with intrapericardial bFGF at $30 \mu \mathrm{g}, 200 \mu \mathrm{g}$, and $2 \mathrm{mg}$. These improvements translated into improved myocardial perfusion and function with histological evidence of increased myocardial vascu- 


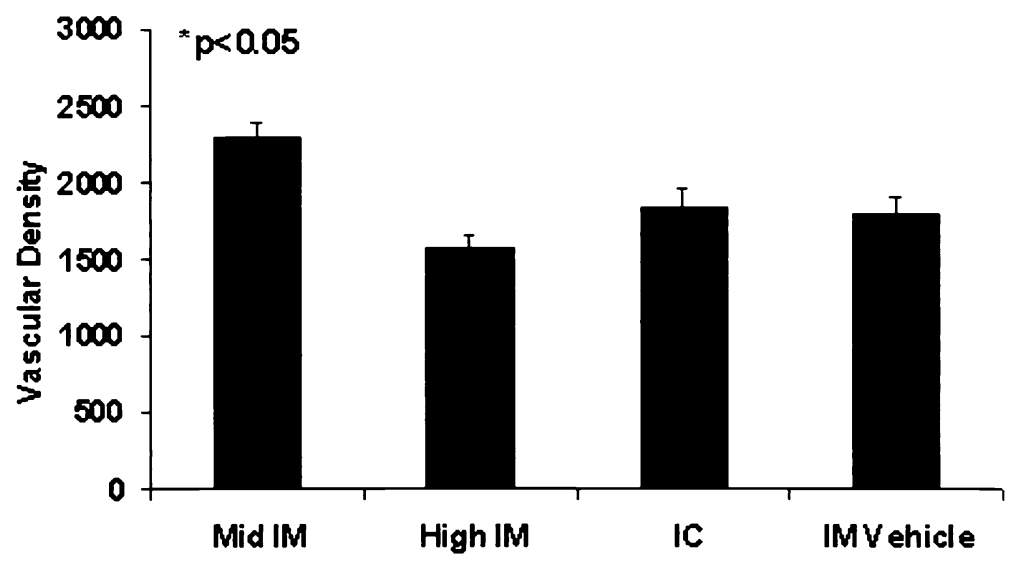

Figure 4. Vascular density analysis. Vascular density counts in each of the $\mathbf{4}$ groups demonstrated a significant increased neovessel count in the mid group when compared to the high, IC, and vehicle controls.

TABLE 5. Serum chemistry data: Hgb, Hct, PIt, and WBC measured at baseline and 2 hours and 3 months following treatment

\begin{tabular}{|c|c|c|c|c|}
\hline Treatment group & $\mathrm{Hgb}$ & Het & PIt & WBC \\
\hline \multicolumn{5}{|l|}{ High $(n=6)$} \\
\hline Baseline & $10.9 \pm 0.8$ & $36.0 \pm 2$ & $472.2 \pm 64.4$ & $11.3 \pm 0.8$ \\
\hline 2 hours & $9.8 \pm 1.3$ & $32.2 \pm 4.0$ & $354.7 \pm 52.5$ & $13.0 \pm 2.4$ \\
\hline 3 months & $12.1 \pm 1.4$ & $39.0 \pm 4.4$ & $437.3 \pm 45.4$ & $12.6 \pm 1.0$ \\
\hline \multicolumn{5}{|l|}{ Mid $(n=6)$} \\
\hline Baseline & $11.7 \pm 0.5$ & $37.8 \pm 1.7$ & $489.2 \pm 73.8$ & $13.2 \pm 1.0$ \\
\hline 2 hours & $10.4 \pm 0.2$ & $34.0 \pm 0.6$ & $479.7 \pm 56.9$ & $13.2 \pm 1.3$ \\
\hline 3 months & $11.4 \pm 0.6$ & $37.3 \pm 1.5$ & $524.5 \pm 31.1$ & $20.2 \pm 6.5$ \\
\hline \multicolumn{5}{|l|}{ IC $(n=6)$} \\
\hline Baseline & $10.7 \pm 0.1$ & $34.5 \pm 0.3$ & $427.8 \pm 40.1$ & $15.4 \pm 4.8$ \\
\hline 2 hours & $10.7 \pm 0.4$ & $34.3 \pm 0.6$ & $432.3 \pm 37.1$ & $15.7 \pm 4.1$ \\
\hline 3 months & $9.9 \pm 0.9$ & $32.7 \pm 3$ & $411.7 \pm 99.8$ & $12.3 \pm 3.4$ \\
\hline \multicolumn{5}{|l|}{ Vehicle $(n=6)$} \\
\hline Baseline & $10.3 \pm 0.4$ & $33.0 \pm 1.3$ & $442.5 \pm 7.8$ & $14.3 \pm 1.4$ \\
\hline 2 hours & $10.4 \pm 0.4$ & $33.5 \pm 1.3$ & $408.8 \pm 13.7$ & $14.9 \pm 1.7$ \\
\hline 3 months & $11.6 \pm 0.2$ & $36.7 \pm 0.6$ & $337.0 \pm 17.2$ & $10.8 \pm 1.8$ \\
\hline
\end{tabular}

IC, Intracoronary. Values reported as the mean \pm standard error of the mean.

larity without any adverse effects 4 weeks after treatment. ${ }^{25}$ Left atrial delivery of $1.74 \mathrm{mg}$ bFGF in canines resulted in a faster improvement in collateral circulation flow that was accompanied by an increase in neovascularization up to 4 weeks after treatment. However, no functional benefits were demonstrated. ${ }^{15}$ Lastly, Rajanayagam and colleagues ${ }^{26}$ utilized in canines 4 routes of delivery of bFGF (central venous injection $1740 \mu \mathrm{g} / \mathrm{d}$ for 1,2 , or 7 days; intravenous infusion $100 \mu \mathrm{g} / \mathrm{kg} / \mathrm{d}$ for 7 days; pericardial instillation $2000 \mu \mathrm{g} / \mathrm{d}$ for 7 days; and intracoronary injection 100 $\mu \mathrm{g} / \mathrm{kg} / \mathrm{d}$ for 1 or 2 days). Using microspheres, maximal collateral perfusion with intracoronary infusion for 2 days exceeded that of control dogs by $31 \%$. Perfusion was not increased following single-dose intracoronary bFGF, and central venous, intravenous, and pericardial injections failed to enhance collateral perfusion. ${ }^{26}$
The ameroid constrictor model remains the most widely used animal model of chronic myocardial ischemia in examining changes in perfusion and function following the administration of angiogenic growth factors, as discussed above. These constrictors are commonly used in swine because of their coronary anatomy, which has minimal preexisting coronary collateral vessels and is similar to human coronary anatomy. Inherent limitations to the use of these occluders include an inability to control the rate or degree of coronary occlusion, which sometimes results in complete vascular occlusion. ${ }^{27-29}$ Also, there is a large variation in the reported percent infarction of the area at risk (5\% to $100 \%){ }^{30}$ Myocardial blood flow and function at rest after ameroid-induced coronary occlusion have been demonstrated to be normal by some investigators due to extensive coronary collateral development. ${ }^{30,31}$ Although the 
TABLE 6. $\mathrm{Na}, \mathrm{K}, \mathrm{Cl}, \mathrm{CO}_{2}, \mathrm{BUN}, \mathrm{Cr}$, and glucose measured at baseline and 2 hours and 3 months following treatment

\begin{tabular}{|c|c|c|c|c|c|c|c|}
\hline Group & $\mathrm{Na}$ & K & CI & $\mathrm{CO}_{2}$ & BUN & $\mathrm{Cr}$ & Glucose \\
\hline \multicolumn{8}{|l|}{ High } \\
\hline Baseline & $137.5 \pm 1.3$ & $4.3 \pm 0.3$ & $98.3 \pm 0.6$ & $32.3 \pm 0.8$ & $10.5 \pm 1.3$ & $1.3 \pm 0.1$ & $68.5 \pm 8.2$ \\
\hline 2 hours & $139.2 \pm 1.8$ & $3.9 \pm 0.3$ & $98.6 \pm 1.8$ & $32.8 \pm 1.3$ & $10.2 \pm 1.0$ & $1.2 \pm 0.1$ & $93.2 \pm 6.1^{*}$ \\
\hline 3 months & $142.5 \pm 1.6$ & $3.9 \pm 0.2$ & $104.2 \pm 1.4$ & $26.8 \pm 2.5$ & $11.6 \pm 3.6$ & $1.5 \pm 0.1$ & $67.0 \pm 9.5$ \\
\hline \multicolumn{8}{|l|}{ Mid } \\
\hline Baseline & $138.5 \pm 1.3$ & $4.9 \pm 0.2$ & $98.7 \pm 1$ & $31.5 \pm 0.7$ & $11.3 \pm 2.0$ & $1.5 \pm 0.1$ & $71.8 \pm 3.9$ \\
\hline 2 hours & $138.2 \pm 0.9$ & $4.8 \pm 0.3$ & $98.8 \pm 1.8$ & $31.2 \pm 1.5$ & $10.0 \pm 1.7$ & $1.3 \pm 0.1$ & $73.8 \pm 6.2$ \\
\hline 3 months & $140 \pm 1.9$ & $4.5 \pm 0.2$ & $98.2 \pm 1.6$ & $26.8 \pm 0.6$ & $9.0 \pm 0.9$ & $1.5 \pm 0.3$ & $79.0 \pm 7.4$ \\
\hline \multicolumn{8}{|l|}{ IC } \\
\hline Baseline & $140.0 \pm 1.5$ & $4.5 \pm 0.5$ & $99.5 \pm 0.9$ & $32.3 \pm 1.9$ & $11.5 \pm 0.3$ & $1.5 \pm 0.2$ & $78.0 \pm 4.4$ \\
\hline 2 hours & $139.0 \pm 2.1$ & $3.6 \pm 0.3$ & $98.5 \pm 0.7$ & $33.0 \pm 1.9$ & $11.5 \pm 0.3$ & $1.5 \pm 0.1$ & $75.8 \pm 4.6$ \\
\hline 3 months & $139.3 \pm 0.7$ & $4.7 \pm 0.6$ & $98.6 \pm 0.9$ & $30.6 \pm 1.0$ & $10.6 \pm 1.7$ & $1.6 \pm 0.1$ & $74.0 \pm 7$ \\
\hline \multicolumn{8}{|l|}{ Vehicle } \\
\hline Baseline & $137.7 \pm 1.1$ & $4.2 \pm 0.2$ & $96.5 \pm 0.6$ & $35.2 \pm 1.3$ & $8.7 \pm 1.8$ & $1.2 \pm 0.1$ & $69.2 \pm 6.1$ \\
\hline 2 hours & $137.0 \pm 1.4$ & $4.7 \pm 0.4$ & $96.0 \pm 1.0$ & $34.5 \pm 1.5$ & $8.2 \pm 1.3$ & $1.1 \pm 0.1$ & $76.5 \pm 6.9$ \\
\hline 3 months & $140.5 \pm 0.7$ & $4.4 \pm 0.2$ & $100.8 \pm 1.1$ & $30.3 \pm 0.7$ & $9.0 \pm 1.2$ & $1.6 \pm 0.1$ & $83.3 \pm 8.1$ \\
\hline
\end{tabular}

$I C$, Intracoronary. Values reported as the mean \pm standard error of the mean.

${ }^{*} P=.04$ by 1 -way repeated measures ANOVA.

TABLE 7. CK, CK/MB, and troponin $\mathrm{T}$ measured at baseline and 2 hours and 3 months following treatment

\begin{tabular}{|c|c|c|c|}
\hline Treatment group & CK & CK-MB & Troponin $\mathrm{T}$ \\
\hline \multicolumn{4}{|l|}{ High $(n=6)$} \\
\hline Baseline & $621.3 \pm 281.2$ & $4.0 \pm 0$ & $0.01 \pm 0.1$ \\
\hline 2 hours & $2532.6 \pm 1263.7$ & $4.7 \pm 0.2$ & $0.02 \pm 0.1$ \\
\hline 3 months & $857.0 \pm 36.8$ & $5.6 \pm 1.3$ & $0.02 \pm 0.1$ \\
\hline \multicolumn{4}{|l|}{ Mid $(n=6)$} \\
\hline Baseline & $2663.5 \pm 872.0$ & $3.8 \pm 0.6$ & $0.01 \pm 0.1$ \\
\hline 2 hours & $1710.5 \pm 422.1$ & $3.7 \pm 0.8$ & $0.02 \pm 0.1$ \\
\hline 3 months & $756.5 \pm 458.7$ & $3.5 \pm 0.6$ & $0.01 \pm 0.1$ \\
\hline \multicolumn{4}{|l|}{$I C(n=6)$} \\
\hline Baseline & $677.0 \pm 238.4$ & $6.3 \pm 3.9$ & $0.01 \pm 0.1$ \\
\hline 2 hours & $888.0 \pm 441.5$ & $6.0 \pm 3.0$ & $0.01 \pm 0.1$ \\
\hline 3 months & $1553.8 \pm 918.5$ & $12.7 \pm 9.1$ & $2.6 \pm 2.6$ \\
\hline \multicolumn{4}{|l|}{ Vehicle $(n=6)$} \\
\hline Baseline & $3744.5 \pm 1360.5$ & $2.8 \pm 0.3$ & $0.01 \pm 0.1$ \\
\hline 2 hours & $4021.0 \pm 1327.1$ & $2.5 \pm 0.5$ & $0.01 \pm 0.1$ \\
\hline 3 months & $602.2 \pm 151.2$ & $2.7 \pm 0.3$ & $0.01 \pm 0.1$ \\
\hline
\end{tabular}

$C K$, Creatine kinase; $C K-M B$, creatine kinase MB isoenzyme; $I C$, intracoronary. Values reported as the mean \pm standard error of the mean.

ameroid model is generally accepted as appropriate for short-term investigations of ischemic myocardium, our partial occlusion model of hibernating myocardium overcomes many of the limitations of the ameroid model. For instance, with our model we are able to control both the rate and degree of coronary occlusion. Furthermore, we have been able to validate the presence of resting myocardial ischemia with viability in the left circumflex artery distribution, with only a small $8 \%$ degree of subendocardial infarction. ${ }^{16}$ Thus, our model is well-suited for studies that involve a longer time period between treatment of ischemic myocardium by angiogenic mechanisms and follow-up functional assessment. For example, our model has been able to dem- onstrate improved myocardial blood flow and improved wall motion 6 months following transmyocardial laser revascularization (TMR). ${ }^{32}$ Our current study was able to demonstrate a similar reversibility of stress-induced ischemia at 3 months.

Intramyocardial mid-dose bFGF may be a novel therapeutic option for end-stage coronary artery disease. Both experimentally and clinically, bFGF doses have ranged from $4 \mu \mathrm{g}$ to upward of 14-mg total dose for ischemic heart disease. Systemic administration of bFGF in amounts necessary to achieve therapeutic myocardial angiogenesis requires large, often daily injections with the potential for systemic toxicity including profound hypotension, hemato- 
TABLE 8. Heart rate and systolic blood pressure at baseline and following treatment (immediately, after 2 hours, and 3 months)

\begin{tabular}{|c|c|c|c|c|}
\hline & High $(n=6)$ & Mid $(n=6)$ & IC $(n=6)$ & Vehicle $(n=6)$ \\
\hline \multicolumn{5}{|l|}{ Heart rate } \\
\hline Baseline & $103.5 \pm 3.5$ & $97.4 \pm 7.8$ & $109.8 \pm 4.4$ & $96.0 \pm 5.6$ \\
\hline Immediate & $86.0 \pm 7.1$ & $100.8 \pm 7.8$ & $124.3 \pm 4.9$ & $78.8 \pm 4.8$ \\
\hline 2 hours & $93.0 \pm 4.1$ & $87.2 \pm 2.2$ & $115.3 \pm 9.7$ & $69.8 \pm 3.6$ \\
\hline 3 months & $117.8 \pm 13.4$ & $116.5 \pm 14.1$ & $108.0 \pm 9.9$ & $111.8 \pm 5.3$ \\
\hline \multicolumn{5}{|l|}{ SBP } \\
\hline Baseline & $75.3 \pm 6.5$ & $88.3 \pm 10.4$ & $117.7 \pm 7.9$ & $81.0 \pm 8.2$ \\
\hline Immediate & $68.5 \pm 7.1$ & $75.0 \pm 17.0$ & $113.5 \pm 4.7$ & $87.5 \pm 8.5$ \\
\hline 2 hours & $77.0 \pm 5.6$ & $63.8 \pm 5.8$ & $119.3 \pm 4.4$ & $78.5 \pm 7.0$ \\
\hline 3 months & $115.0 \pm 15.5$ & $105.5 \pm 8.9$ & $114.3 \pm 6.7$ & $95.2 \pm 1.9$ \\
\hline
\end{tabular}

$I C$, Intracoronary; $S B P$, systolic blood pressure. Values are reported as the mean \pm standard error of the mean. $\mathrm{HR}$ in beats/minute; $\mathrm{SBP}$ in $\mathrm{mm} \mathrm{Hg}$.

TABLE 9. Serum bFGF-2 levels at baseline and 3 months following treatment

\begin{tabular}{lcc}
\hline Treatment group & Baseline bFGF-2 & 3 month bFGF-2 \\
\hline High $(\mathrm{n}=6)$ & $133.3 \pm 48.5$ & $203.0 \pm 89.0$ \\
Mid $(\mathrm{n}=6)$ & $62.3 \pm 32.7$ & $61.6 \pm 28.5$ \\
IC $(\mathrm{n}=6)$ & $2.6 \pm 1.4$ & $18.1 \pm 6.0^{*}$ \\
Vehicle $(\mathrm{n}=6)$ & $46.7 \pm 35.0$ & $7.5 \pm 4.8$ \\
\hline
\end{tabular}

$\overline{b F G F}$, Basic fibroblast growth factor 2; IC, intracoronary. Values are reported as the mean \pm standard error of the mean, in $\mathrm{pg} / \mathrm{mL}$.

${ }^{*} P=.038$ by 1 -way repeated measures ANOVA.

logical, and renal end-organ toxicity. There has been concern about potential acceleration of occult neoplastic disease or diabetic retinopathy with the use of angiogenic growth factors. In addition, the mitogenic effects of angiogenic growth factors (mostly vascular endothelial growth factor) may accelerate atherosclerotic lesion expansion and/or may lead to plaque destabilization, as demonstrated by a series of investigators. ${ }^{33,34}$ Lastly, administration of bFGF in high dosages is also associated with numerous side effects including anemia, thrombocytopenia, membranous nephropathy, and hyperostosis. ${ }^{35}$

Though we did not examine specific end-organ toxicity, local administration of very low doses of bFGF into the myocardium may provide the safest means of delivery of a potentially toxic medication. The main strength of the current study is the long time period between treatment with bFGF and follow-up measurement of PET and DSE. To our knowledge, no other investigator has demonstrated the effects of exogenous growth factor administration as long as 3 months after treatment. Despite this strength, we were not able to show that the increased myocardial perfusion found after mid- or high-dose intramyocardial bFGF therapy translates into improved systolic function. Perhaps an even longer follow-up period may be required to show such benefit. We have also demonstrated a potential link between capillary density and increased regional perfusion, as was demonstrated in the mid-dose IM bFGF group. Thus, the neovascularization that results from exogenous angiogenin administration may be directly responsible for the improvement in regional perfusion found after such intervention.

The main limitation of our study, however, was our inability to show this link between improved capillary density and increased myocardial perfusion in the high-dose IM bFGF group. One potential reason for this inconsistency may have been the random tissue sampling technique used in our vascular density analysis.

In summary, we have shown that high- and mid-dose bFGF-2 delivered by direct intramyocardial injection results in significant improvement in perfusion of chronically ischemic porcine myocardium 3 months later. Meanwhile, intracoronary infusion of this growth factor does not appear to significantly augment such perfusion. The increase in myocardial blood flow achieved by IM bFGF did not result in improved contractile function of that myocardium, indicating that the functional benefits of bFGF therapy may require longer time periods to develop.

\section{References}

1. Symes J, Losorodo D, Vale P, et al. Gene therapy with vascular endothelial growth factor for inoperable coronary artery disease. Ann Thorac Surg. 1999;68:830-7.

2. Henry T. Therapeutic angiogenesis. BMJ. 1999;318:1536-40.

3. Hockel MSK, Doctrow S, Kissel T, Vaupel P. Therapeutic angiogenesis. Arch Surg. 1993;128:423-9.

4. Folkman J, Shing Y. Angiogenesis. J Biol Chem. 1992;267:10931-4.

5. Yoshiji H, Gomez DE, Shibuya M, Thorgeirsson UP. Expression of vascular endothelial growth factor, its receptor, and other angiogenic factors in human breast cancer. Cancer Res. 1996;56:2013-6.

6. Paques M, Massin P, Gaudric A. Growth factors and diabetic retinopathy. Diabetes Metab. 1997;23:125-30.

7. Favard C, Ortega N, Bayard F, Plouet J. Vascular endothelial growth factor and retinal neovascularization: a new therapeutic approach for diabetic retinopathy. Diabetes Metab. 1996;22:268-73.

8. Samoto K, Ikezaki K, Ono M, et al. Expression of vascular endothelial growth factor and its possible relation with neovascularization in human brain tumors. Cancer Res. 1995;55:1189-93.

9. Aiello LP, Avery RL, Arrigg PG, et al. Vascular endothelial growth factor in ocular fluid of patients with diabetic retinopathy and other retinal disorders. $N$ Engl J Med. 1994;331:1480-7. 
10. Boulton M, Gregor Z, McLeod D, et al. Intravitreal growth factors in proliferative diabetic retinopathy: correlation with neovascular activity and glycaemic management. Br J Ophthalmol. 1997;81:228-33.

11. Harada K, Grossman W, Friedman M, et al. Basic fibroblast growth factor improves myocardial function in chronically ischemic porcine hearts. J Clin Invest. 1994;94:623-30.

12. Lopez JJ, Edelman ER, Stamler A, et al. Basic fibroblast growth factor in a porcine model of chronic myocardial ischemia: a comparison of angiographic, echocardiographic and coronary flow parameters. $J$ Pharmacol Exp Ther. 1997;282:385-90.

13. Unger EF, Banai S, Shou M, et al. Basic fibroblast growth factor enhances myocardial collateral flow in a canine model. Am J Physiol. 1994;266:H1588-95.

14. Laham RJ, Hung D, Simons M. Therapeutic myocardial angiogenesis using percutaneous intrapericardial drug delivery. Clin Cardiol. 1999; 22:I6-9.

15. Lazarous DF, Shou M, Stiber JA, et al. Pharmacodynamics of basic fibroblast growth factor: route of administration determines myocardial and systemic distribution. Cardiovasc Res. 1997;36:78-85.

16. St Louis J, Hughes G, Kypson A, et al. An experimental model of chronic myocardial hibernation. Ann Thorac Surg. 2000;69:1351-7.

17. Camici P, Ferrannini E, Opie L. Myocardial metabolism in ischemic heart disease: basic principles and application to imaging by positron emission tomography. Prog Cardiovasc Dis. 1989;32:217-38.

18. Cigarroa C, deFilippi C, Brickner M, Alvarez L, Wait M, Grayburn P. Dobutamine stress echocardiography identifies hibernating myocardium and predicts recovery of left ventricular function after coronary revascularization. Circulation. 1993;88:430-6.

19. Afridi I, Kleiman N, Raizner A, Zoghbi W. Dobutamine echocardiography in myocardial hibernation: optimal dose and accuracy in predicting recovery of ventricular function after coronary angioplasty. Circulation. 1995;91:663-70.

20. DeGrado T, Hanson M, Turkington T, et al. Estimation of myocardial blood flow for longitudinal studies with $13 \mathrm{~N}$-labeled ammonia and positron emission tomography. J Nucl Cardiol. 1996;3:494-507.

21. Gambhir S, Schwaiger M, Huang S, et al. Simple noninvasive quantification method for measuring myocardial glucose utilization in humans employing positron emission tomography and fluorine-18 deoxyglucose. J Nucl Med. 1989;30:359-66.

22. Ng C, Holden J, DeGrado T, Raffel D, Kornguth M, Gatley SJ. Sensitivity of myocardial fluorodeoxyglucose lumped constant to glucose and insulin. Am J Physiol. 1991;260:H593-603.

23. Rahimtoola S. Hibernating myocardium has reduced blood flow at rest that increases with low-dose dobutamine. Circulation. 1996;94:305561.

24. Takeshita SZL, Brogi E, Kearney M, et al. Therapeutic angiogenesis. A single intraarterial bolus of vascular endothelial growth factor augments revascularization in a rabbit ischemic hind limb model. J Clin Invest. 1994;93:662-70.

25. Laham RJ, Rezaee M, Post M, et al. Intrapericardial delivery of fibroblast growth factor-2 induces neovascularization in a porcine model of chronic myocardial ischemia. J Pharmacol Exp Ther. 2000; 292:795-802.

26. Rajanayagam MAS, Shou M, Thirumurti V, et al. Intracoronary basic fibroblast growth factor enhances myocardial collateral perfusion in dogs. JACC. 2000;35:519-26.

27. Bernotat-Danielowski S, Sharma H, Schott R, Schaper W. Generation and localization of monoclonal antibodies against fibroblast growth factor in ischaemic collateralized porcine myocardium. Cardiovasc Res. 1993;27:1220-8.

28. Inou $\mathrm{T}$, Tomoioke $\mathrm{H}$, Watanabe $\mathrm{K}$, et al. A newly developed $\mathrm{x}$-ray transparent ameroid constrictor for study on progression of gradual coronary stenosis. Basic Res Cardiol. 1980;75:537-43.

29. Shen Y-T, Vatner S. Mechanism of impaired myocardial function during progressive coronary stenosis in conscious pigs. Hibernation versus stunning? Circ Res. 1995;76:479-88.

30. O'Konski M, Roth D, White F. Ameroid constriction of the proximal left circumflex coronary artery in swine. Am J Cardiovasc Pathol. 1986;1:69-77.

31. Roth D, Maruoka Y, Rogers J, White F, Longhurst J, Bloor C. Development of coronary collateral circulation in left circumflex ameroid-occluded swine myocardium. Am J Physiol. 1987;253:H1279-288.

32. Hughes GC, Lowe JE, Kypson AP, et al. Neovascularization after transmyocardial laser revascularization in a model of chronic ischemia. Ann Thorac Surg. 1998;66:2029-36.

33. Flugelman MY, Virmani R, Correa R, et al. Smooth muscle cell abundance and fibroblast growth factors in coronary lesions of patients with nonfatal unstable angina. A clue to the mechanism of transformation from the stable to the unstable clinical state. Circulation. 1993;88:2493-500.

34. Nabel EG, Yang ZY, Plautz G, et al. Recombinant fibroblast growth factor-1 promotes intimal hyperplasia and angiogenesis in arteries in vivo. Nature. 1993;362:844-6.

35. Mazue G, Bertolero F, Jacob C, Sarmientos P, Roncucci R. Preclinical and clinical studies with recombinant human basic fibroblast growth factor. Ann N Y Acad Sci. 1991;638:329-40. 